\title{
Smart City Waste Management through ICT and IoT driven Solution
}

\author{
Dipak S. Gade ${ }^{1} \&$ P. S. Aithal ${ }^{2}$ \\ ${ }^{1}$ Post Doctoral Research Scholar, Department of Computer Science and Engineering, \\ Srinivas University, Mangalore, India \\ ORCID ID: 0000-0002-0962-0375, Email: dipak.pdf@ srinivasuniversity.edu.in \\ ${ }^{2}$ Vice Chancellor, Srinivas University, Mangalore, India \\ ORCID ID: 0000-0002-4691-8736, E-mail: psaithal@ gmail.com
}

Subject Area: Information Technology.

Type of the Paper: Prototype and Simulation based Research.

Type of Review: Peer Reviewed as per $|\mathrm{C}| \mathrm{O}|\mathrm{P}| \mathrm{E} \mid$ guidance.

Indexed In: OpenAIRE.

DOI: http://doi.org/10.5281/zenodo.4739109

Google Scholar Citation: IJAEML

\section{How to Cite this Paper:}

Gade, Dipak S., \& Aithal, P. S., (2021). Smart City Waste Management through ICT and IoT driven Solution. International Journal of Applied Engineering and Management Letters (IJAEML), 5(1), 51-65. DOI: http://doi.org/10.5281/zenodo.4739109.

International Journal of Applied Engineering and Management Letters (IJAEML)

A Refereed International Journal of Srinivas University, India.

Crossref DOI : $\underline{\text { https://doi.org/10.47992/IJAEML.2581.7000.0092 }}$

(C) With Authors.

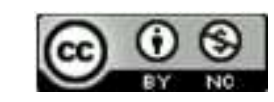

This work is licensed under a Creative Commons Attribution-Non-Commercial 4.0 International License subject to proper citation to the publication source of the work.

Disclaimer: The scholarly papers as reviewed and published by the Srinivas Publications (S.P.), India are the views and opinions of their respective authors and are not the views or opinions of the S.P. The S.P. disclaims of any harm or loss caused due to the published content to any party. 


\title{
Smart City Waste Management through ICT and IoT driven Solution
}

\author{
Dipak S. Gade ${ }^{1} \&$ P. S. Aithal ${ }^{2}$ \\ ${ }^{1}$ Post Doctoral Research Scholar, Department of Computer Science and Engineering, \\ Srinivas University, Mangalore, India \\ ORCID ID: 0000-0002-0962-0375, Email: dipak.pdf@ srinivasuniversity.edu.in \\ ${ }^{2}$ Vice Chancellor, Srinivas University, Mangalore, India \\ ORCID ID: 0000-0002-4691-8736, E-mail: psaithal@ gmail.com
}

\begin{abstract}
Purpose: The growing population and mass relocation of citizens from urban and semi-urban areas to Smart Cities have resulted in exponential growth in Smart Cities and thereby certain challenges. One of the major challenges Smart Cities are facing is to control, manage and process waste generation on a daily basis. Waste collection and processing at a wider scale is not an easy job. The growing population and resource constraints in waste management activities are the primary reasons, which have made waste management a tough job. To deal with this challenging process, Smart Cities use Smart Waste Management System. This paper has provided an overview of a typical Smart Waste Management system and a review of selected research papers on Smart Waste Management. We tried to identify areas of improvement with existing Smart Waste Management Solutions and proposed an innovative solution called "iSmartWMS" for carrying out waste management specifically for Smart Cities. The paper has discussed in detail the architecture and building blocks of the proposed Smart Waste Management System, along with the details of software tools, sensors, and technologies proposed in iSmartWMS. The Paper has finally discussed results with respect to the prototype implementation of iSmartWMS and also future plans to further improve the iSmartWMS smart waste management system.
\end{abstract}

Design/Methodology/Approach: iSmartWMS software prototype was built using IoT sensors and Cloud based Server running with custom software incorporating specialized algorithms and a graphical user interface. A model was simulated on a local machine network to check if the required goals can be met and if the proposed solution serves the purpose.

Findings/Result: The proof of concept prototype for iSmartWMS Solution is found working well at a limited scale. It is clear that the solution can very well serve the purpose of waste management if it is implemented as per specified architecture at wider scale considering the large number of stakeholders.

Originality/Value: Using IoT Sensors for waste monitoring and through Cloud based Server software running with specialized algorithms, it is possible to automate waste management end to end activities. This paper has described in detail the proposed iSmartWMS software solution as a Smart Waste Management tool for Smart Cities.

Paper Type: Prototype and Simulation based Research

Keywords: Solid Waste Management, Smart Waste Bin, IoT, Sensor Fusion, Image Processing, Data Analytics

\section{INTRODUCTION :}

Ever growing population causing a lot of issues and one of the biggest issues is increasing generated waste. Such waste can be food waste, material waste, waste from human beings, waste from useless stuff, waste from industries, etc. Such generated waste is hazardous for the overall environment if not managed systematically. Hence waste management is a very important issue that requires high attention and resolution on priority. 


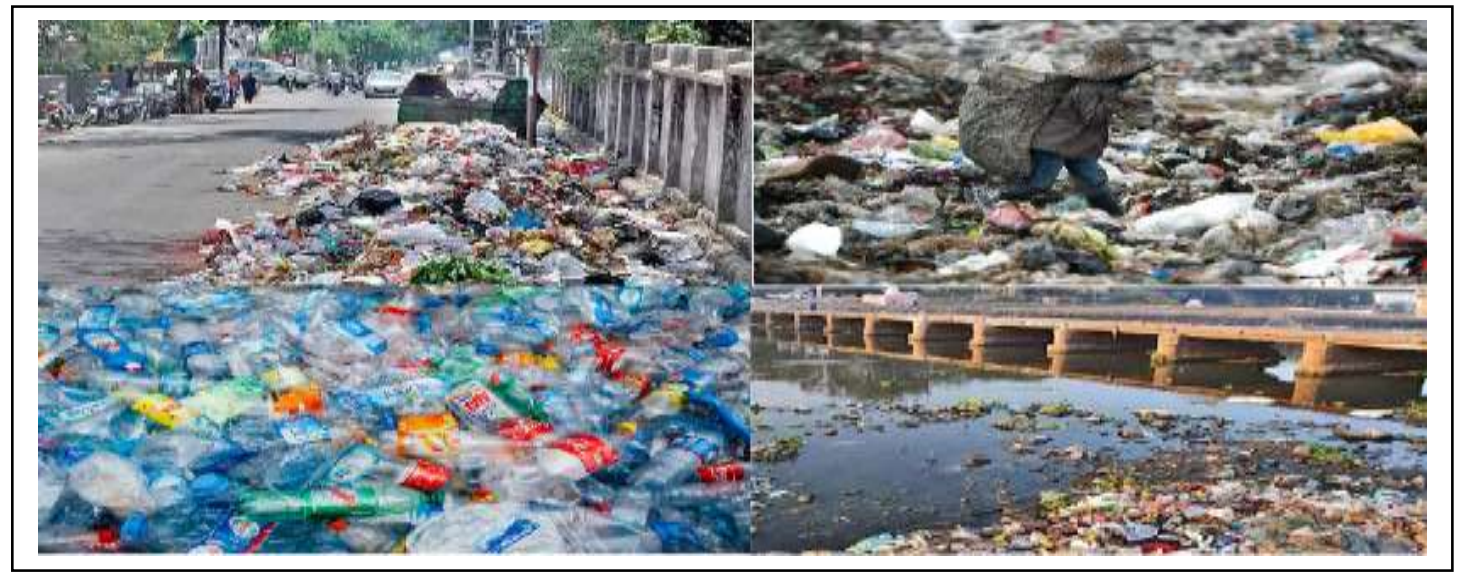

Fig. 1: Unattended deposited waste in water, ground and on road [1]

The major contribution for waste generation is from human activities [1]. It should be noted that waste management's aim is not just disposing of the waste in the appropriate manner but also to control, collect, process, and recycle it. Waste management covers end-to-end activities involved such as monitoring the generated waste in City, from its source when citizens produce waste through waste collection, waste transportation, and depositing it to waste depo which can then be the landfill, incineration, and or recycled [2]. In Waste Management activities, prevention of waste is considered as the preferred option followed by reusing the material, recycling of the material, generating energy through waste, and finally waste disposal is considered as the least preferred alternative [3]. There are a variety of ways by which waste can be controlled, disposed of, processed, and recycled into useful stuff. Today waste management is also becoming challenging due to the enormous increase in the volume of generated waste. High volume generated waste requires larger manpower and larger efforts and increased cost in handling waste management activities. Refer to figure 1 which has shown high volume deposited waste in water and on the ground. In many cities and rural areas due to the absence of waste management staff and system in place, waste is regularly abandoned in open pit or on empty ground, or even in river water. Such openly accumulated waste subsequently becomes solid and converts into a waste heap and starts generating foul smell and toxic gases which are very dangerous to human health as well as hazardous to the environment.

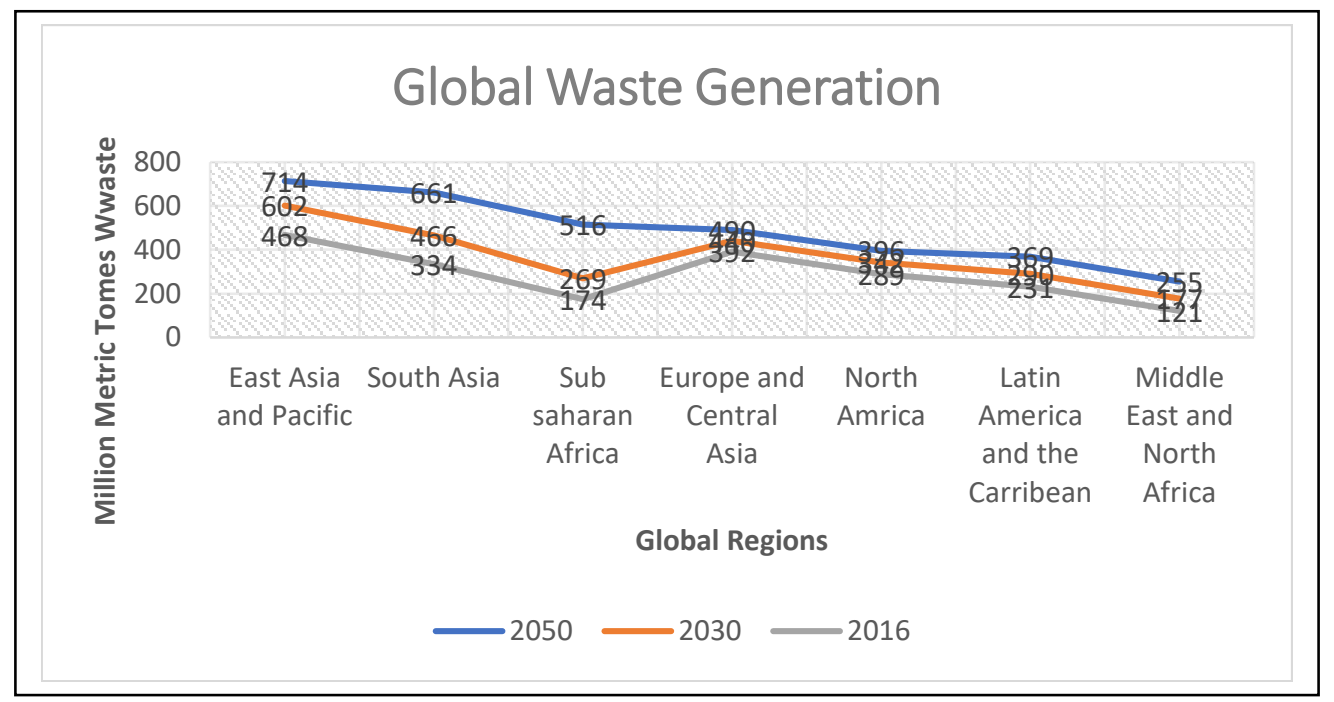

Fig. 2: Global Waste generation projection [4]

Refer to figure 2 which has shown the waste generation forecast in million metric tonnes for various regions across the globe for the period of 2016 to 2050, as per the statista.com projection. Due to growing populations and rapid urbanization, local annual waste generation is expected to jump to 3.4 billion tonnes over the next few decades, according to the World Bank Group. By 2050, it is estimated 
that around 714 million metric tonnes of waste will be generated alone in East Asia and the Pacific region [4]. It is scary to know that waste generation is going to increase exponentially over the period of time and is an alarming situation. We need to take the right action now. This is the time to control and dispose of the generated waste as effectively as possible so that the possible hazardous impact on the environment and human being health can be effectively reduced. Before further deep dive, let us understand what the different types of waste are [5] which need proper management.

(1) Organic Waste: It is the garbage derived from organic material.

(2) Industrial Waste: It is the residual solid waste generated from industrial production.

(3) Hospital Waste: It is the waste generating at hospitals and or medical clinics due to medical operations, patient tests, and treatments. This kind of waste can be dangerous to human beings and may cause to transmit diseases to people who come in contact with it.

(4) Electronic Waste: It is generated by the disposal of obsolete Electronics Products.

(5) Nuclear Waste: It is generated at Nuclear Plants during plants operation. Such kind of waste can be radioactive and is extremely dangerous for human beings and the environment. Hence, it needs to be disposed of in strict safety guidelines

(6) Commercial Waste: It is generated by commercial establishments including shops, warehouses, appliances stores etc. Such kind of waste can be recycled entirely.

(7) Green Waste: This kind of waste gets generated due to trees. e.g. fallen leaves from trees, fallen fruits, from the pruning of trees, branches, trunks. Since it is mainly organic in nature, such kind of waste can be processed to produce fertilizers

(8) Recyclable Waste: It is typically the waste that gets generated at residences, organization premises. Such kind of waste can be reused after processing and after transformation to other useful stuff.

Traditional waste management often involves the manual collection of waste from waste bins and its manual disposal or recycling through a defined process [6]. Though this process helps, it involves a lot of human efforts and significant errors due to manually handled activities. Often these activities and efforts made are found inefficient specifically when the amount of generated waste is higher. Besides, it also creates other problems such as

- During waste collection, it is possible that some of the waste bins might be overfilled and some might be underfilled

- Overfilled waste bins can create toxic gases and foul smell and thus can create unhygienic conditions

- Waste collection from underfilled the waste bins can create inefficiency in the waste collection process

- Waste collection vehicles typically collect the waste in sequence irrespective of the waste fill status of the waste bins. These unoptimized routes can result in excessive fuel consumption and environmental pollution

- Combining collected waste at the central point, can complicate waste sorting and recycling operation

- Also due to not applying relevant tools and technologies, the waste management process becomes very difficult and inefficient.

\section{PROBLEM STATEMENT :}

Smart Cities are rapidly growing and globally set up by various countries for providing world-class services and enjoyable life to their residents. This has attracted many citizens to Smart Cities by leaving their existing cities and hometowns. This has resulted in exponential growth and expansion of Smart Cities at the same time multi-fold problems like an overload on Smart Cities to handle the growing population and to effectively control the waste produced by the huge population, let it be human beings made waste, industrial waste, environmental waste and or medical waste. If a large amount of generated waste is not handled correctly and efficiently, it can generate a hazardous situation and can cause danger to a great extent to the Smart City Residents. Also, a shortfall can be possible with some of the necessary items which go to waste after initial use, and if we do not recycle them, such items cost gets increases 
over time due to shortfall in supply and causes impacting the economy.

If we can find out a way to ensure safe and efficient waste management with low cost and low processing time, it will not only help to maintain healthy surroundings but also by recycling reusable waste, it can easily boost the economy and manage the shortfall of necessary items such as paper, footwear, stationery items, tyres, remouldable plastic and so on.

\section{RESEARCH OBJECTIVES :}

Research Area related to Waste Management Solutions is vast and time-consuming. With the available time constraints and limited infrastructure, research is carried out keeping in mind the following objectives

- Study the existing Waste Management Solutions of Smart Cities

- Identify deficiencies, limitations, and issues if any with the existing Waste Management Solutions deployed in various Smart Cities.

- Propose the IoT and ICT based solution to effectively carry out waste management specifically for Smart Cities

- Identify the improvement opportunities which can be carried out in future for the proposed Waste Management Solution.

\section{METHODOLOGY :}

While conducting the research and proposing a new solution for Waste Management, multiple methodologies and techniques were used by us. The main focus was on carrying out a time-bound existing literature review. For this purpose, we studied the relevant research papers, journal papers, white papers, conference papers, online blogs, and dedicated websites for Smart Waste Management. Specific keywords such as "Smart City", "Smart Waste Management" "Waste Handling", "Waste Generation", "Waste Recycling" were used while identifying relevant research papers and book chapters. The collected literature was then analysed considering the year it is published, proposed solutions for Smart Waste Management, notes on existing techniques while carrying out waste management across the globe, new considerations in new Smart Cities for waste management and also the operating cost, manpower and required infrastructure for doing the Smart Waste Management. We also went through the existing Interviews of Subject Matter Experts, researchers and industry professionals working in Smart Waste Management. Few Interviews were also taken of some wellknown industrial professional enquiring their opinions and views on existing Waste Management techniques and suggestions for improvements in Smart Waste Management. Last we carried out certain experiments through simulations and prototypes to some extent to build a proof of concepts around the proposed solution (at lab level) to measure its efficiency and effective operations meeting the stated objectives.

\section{RELATED WORKS :}

Due to increasing population, urbanisation and industrial processing, a lot of waste is getting generated day by day. If this generated waste is not processed and managed properly then there are good chances of health hazards, environmental pollution, and contamination [7]. To avoid such issue well ahead of time, waste management is considered a priority task to address for the last few years. Worldwide researchers, scientist and engineers are working on making improvements in existing Smart Waste Management Systems as well as identifying new features and functionalities to incorporate into the new Smart waste Management System being developed. Many research papers and significant reading material is available online on Smart Waste Management System. Let us review some of the existing research papers to get enough insights into this research topic.

Insung Hong et al, in their paper "IoT-Based Smart Garbage System for Efficient Food Waste Management" have proposed an IoT-based Smart Garbage System known as SGS for efficient food waste management [8]. In the SGS Pilot project, authors have used battery-based Smart garbage bins which can exchange information with each other using a wireless mesh network. The router and Server analyze the information exchanged by smart garbage bins and take appropriate actions with respect to food waste. To increase the lifetime of battery used in the SGS system, authors also supported two types of energy-efficient operations with Smart garbage bins, one is standalone operation mode and the second is cooperation-based operation mode. Authors have claimed that after using SGS on a pilot basis 
for a period of one year they found that the average amount of food waste decreased by almost $33 \%$. Today there are a lot of systems that widely use and employ IoT-based sensors for data collection. I feel that the accuracy of collected data through IoT sensors becomes the backbone of such systems and hence it is of utmost importance to ensure that the sensors are working properly and are calibrated on regular basis.

María-Victoria Bueno-Delgado et al, in their paper "Optimal Path Planning for Selective Waste Collection in Smart Cities" have discussed the optimal planning algorithm [9]. Authors have claimed that this optimal planning algorithm with a suitable practical software platform, enables computation of optimal waste collection routes for Smart and sustainable cities. optimal planning algorithm which is executed in an open-source planning tool known as Net2Plan. Net2Plan is typically used for modelling and planning communication networks. Net2Plan along with Net2Plan-GIS library facilitates city layout input information to the algorithm including the positions of smart bins. The computed optimal waste collection paths minimize the number of trucks to be used for waste collection and thereby fuel consumption, this subsequently reduces the impact of $\mathrm{CO} 2$ emissions and acoustic damages. The authors have also discussed a practical case of the city of Cartagena (Spain) where the optimal path planning for plastic waste collection is addressed.

A smart network based on smart sensors which are capable of communicating with any authentic Server which can come within the range of communication is also becoming a convenient concept in view and need wide-area coverage for waste collection and management. This concept is well described by Yann Glouche et al [10], in their paper "A Smart Waste Management with Self-Describing objects". In this paper, the authors have discussed a solution based on RFID Tag which provides information about the waste bins. This information subsequently used to improve waste management by providing early automatic identification of waste at the bin level. Authors have claimed that the waste tracking using Smart Bins equipped with RFID-based Tags, doesn't require any other external information system and this solution enables improvement in the selective sorting of Waste. Besides this RFID Tag helps to report back the waste contents of Smart Bin back to the recycling system.

On the other hand, it is always challenging to analyse the generated data systematically and a need to have a proper query mechanism to filter the right information so that one can easily know how much waste got generated, how much waste is processed, how much waste is recycled and so on. Zeki Oralhan et al, in their paper "Smart City Application: Internet of Things (IoT) Technologies Based Smart Waste Collection Using Data Mining Approach and Ant Colony Optimization", have discussed the design of garbage container integrated with sensors for measurement of temperature within the container, the ratio of $\mathrm{Co} 2$ within the container and fill level of the container [11]. The measured information from the container with the help of IoT Sensors then transmitted to the waste management Software System. The waste management software system then using a data mining approach forecasts when garbage containers can be completely filled and need replacement. Also, by using the ANT colony algorithm the software calculates the most efficient waste collection routes, and this route information is then passed on to waste collection trucks on the smart tablet. Authors have claimed that the developed system when implemented on a pilot basis in a city it offered around 30\% of cost savings in waste collection. Also, this system helped in reducing garbage collection truck's oil costs, carbon emissions, traffic, and other negative environmental impacts.

Today based on the coverage areas, the municipality and Smart Cities Administration department arranges waste collection vehicles to collect the waste from various parts of cities and geographies. This manual collection of waste is considered a time-consuming and tedious job. Imagine how much time it can save if the whole process is automated. Considering the amount of time, it is taking up for waste collection ultimately there is a dire need for automated waste collection. Cicerone Laurentiu Popa et al [12], in their paper, "Smart City Platform Development for an Automated Waste Collection System", have discussed the development of a fully automated Smart waste collection System. Authors have claimed that such a system can be used to convert the automated waste collection system into a smart system and can be fit for use within Smart City Infrastructure. The authors have clarified that they used the Azure platform for the development of the proposed system. This Azure Platform facilitates realtime monitoring and communication with a central system. With the help of IoT-based Sensors, a Cloud Platform, and with data analytics, the Smart Waste management system could achieve fill status information of waste bins, route optimization for Vehicles used for Waste collection, Waste Bin status information, and so on. 


\section{WASTE MANAGEMENT CONCEPTS - DEEP DIVING :}

Considering the significant negative impact due to inefficient waste management, in Smart Cities, waste management activity is given a top priority and often carried out in a controlled manner using the latest tools and technologies such as IoT, data analytics, Client Server, Image Processing, Smart Sensors, etc [13]. Such waste management often carried out right from controlled and efficient collection of waste, waste monitoring, and analysis, waste transportation, waste processing, waste recycling, and finally environment-friendly waste disposal to ensure the minimum negative impact on the environment and Smart City residents. Such kind of waste management is often called Smart Waste Management.

\subsection{Main Elements of Smart Waste Management}

The main technology elements for Smart Waste Management are as follows.

(1) Automatic Alerts for Waste Bins filling Status

(2) Automated Waste Collection

(3) Landfill Modernization

(4) Energy Generation

(5) Environment Friendly Operation

Let us walk through some of the special features and advantages available with Smart Waste Management system.

(1) Automatic Alerts for Waste Bins filling Status

Using variety of Sensors along with Smart Trash bin it is possible to provide alerts and notifications when the waste bin needs to be serviced. This facilitates waste collection vehicles to be deployed to collect waste from only those containers which needs to be emptied.

\section{(2) Automated Waste Collection}

Using Autonomous Vehicles, equipped with Waste Collection Container and Robotic Arm for waste pickup and disposal in waste container, has made the waste collection process truly automatic in practical sense. This not only reduces the overall time required in waste collection but also the efforts and thus increases the overall process efficiency.

\section{(3) Landfill Modernization}

This is possible by modernizing the garbage dumps and highly engineered landfill operations complied with local and state level relevant regulations to reduce emission of methane gas and ensure adequate protection to human health and surrounding environment.

\section{(4) Energy Generation}

Waste may contain different materials such as agricultural leftovers, food, animal waste, etc. which can be suitably processed to produce biogas. With the help of Digesters, it can be possible to covert the biogas into energy. There are Thermal Converters available which helps to convert useless materials into useful stuff. Also, through proper processing of released gases by landfills it is possible to get the appropriate energy with the help of Bioreactors, Fuel cells and Microturbine Technology. Refer figure 3, which has presented typical stages in gas to energy transformation process.

\section{(5) Environment Friendly Operation}

By using the Waste Collection Vehicles running on Natural Gases instead of Petrol or Diesel, the overall transportation is quieter as well as cost effective. Also, timely collection of garbage from the waste bin ensure to avoid spreading of any harmful gases and foul smells in the environment. Besides, timely recycling of the waste also ensures avoiding any chances of depositing any harmful 
material in ground and or surrounding atmosphere. This whole operation finally helps in reducing the carbon footprint and overall negative impact on the environment.

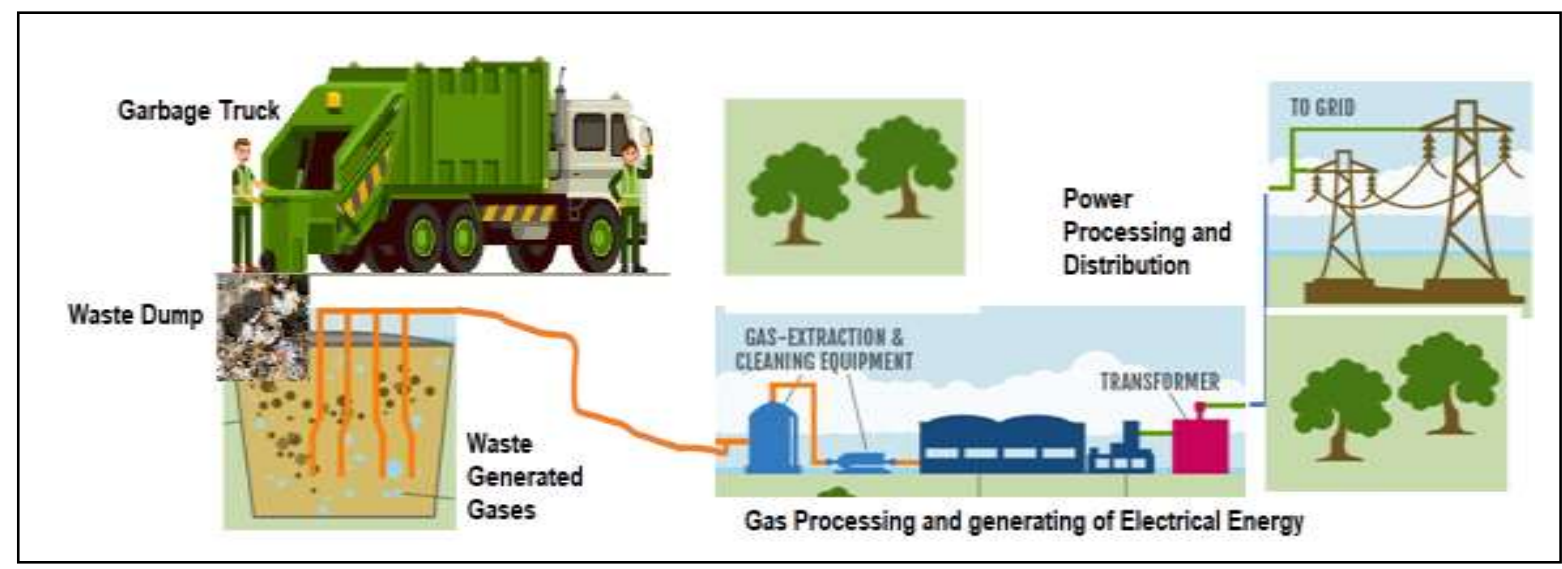

Fig. 3: Solid waste landfill gas-to-energy systems

Overall, Smart Waste Management System facilitates waste collection from waste bins only when required, efficient waste collection and transportation, automated process operations, optimized resource utilization and helps in maintaining the healthy environment.

\section{SMART WASTE MANAGEMENT SYSTEM "ISMARTWMS":}

Based on the key requirements to be addressed while dealing with waste management process and keeping in mind operation efficiency, a cloud based, IoT sensors driven smart solution called as iSmartWMS is proposed for managing end to end waste management process. iSmartWMS is based on secure end to encrypted communication and multi-tier Client Server communication architecture. Please refer figure 4 which has shown high level building blocks of iSmartWMS. Let us walk through the major components of iSmartWMS [14-28].

\section{(1) Smart Trash Bin}

Smart Trash Bins are more than a typical trash bin (also known as garbage bins or waste bins) used to store the waste. Users can deposit the waste from the bin opening conveniently. The trash can store wet and dry solid waste as usual. However, Smart Trash Bins also offer it's fill level information as well as servicing need information to external world. To do this, it employs variety of sensors capable of sensing the relevant things and connecting to the internet network for communication purpose. The popular sensors used in Smart Trash Bin are as follows.

- Ultrasonic Sensors: These sensors measure fill-levels in trash bins via ultrasonic beams. Ultrasonic Sensors can easily set to measure distance from few $\mathrm{cm}$ to few hundred $\mathrm{cm}$. Ultrasonic technology ensures top measurement accuracy. The information from sensors can be communicated using GSM Modems or IoT networks LORA / MQTT / COAP communication protocols. The communication can be encrypted for preventing misuse of information. For Sensor electronic circuit operation, long life replaceable batteries are connected, having typical lifespan of around 2 Years or more. Such sensors need to be light weight, compact, robust, water and shock resistant and supporting wide operational temperature range.

- RFID TAG: The RFID Sensors are very compact and can fit anywhere. The tags are passive contactless transponders which can be installed easily into most waste bins including metal bins and plastic bins However, in plastic bins they really perform well. RFID Tags doesn't need the line of sight for operation. These TAGS are configurable to operate in LF, HF and UHF Band and often fitted with some memory for data storage, read and write purpose. The TAGs can be read from around 5 to $6 \mathrm{ft}$ distance. In addition to service verification and route management of waste vehicles, RFID Tags fitted with Trash bins helps in trash bins tracking, repairs of waste containers and work order management. 


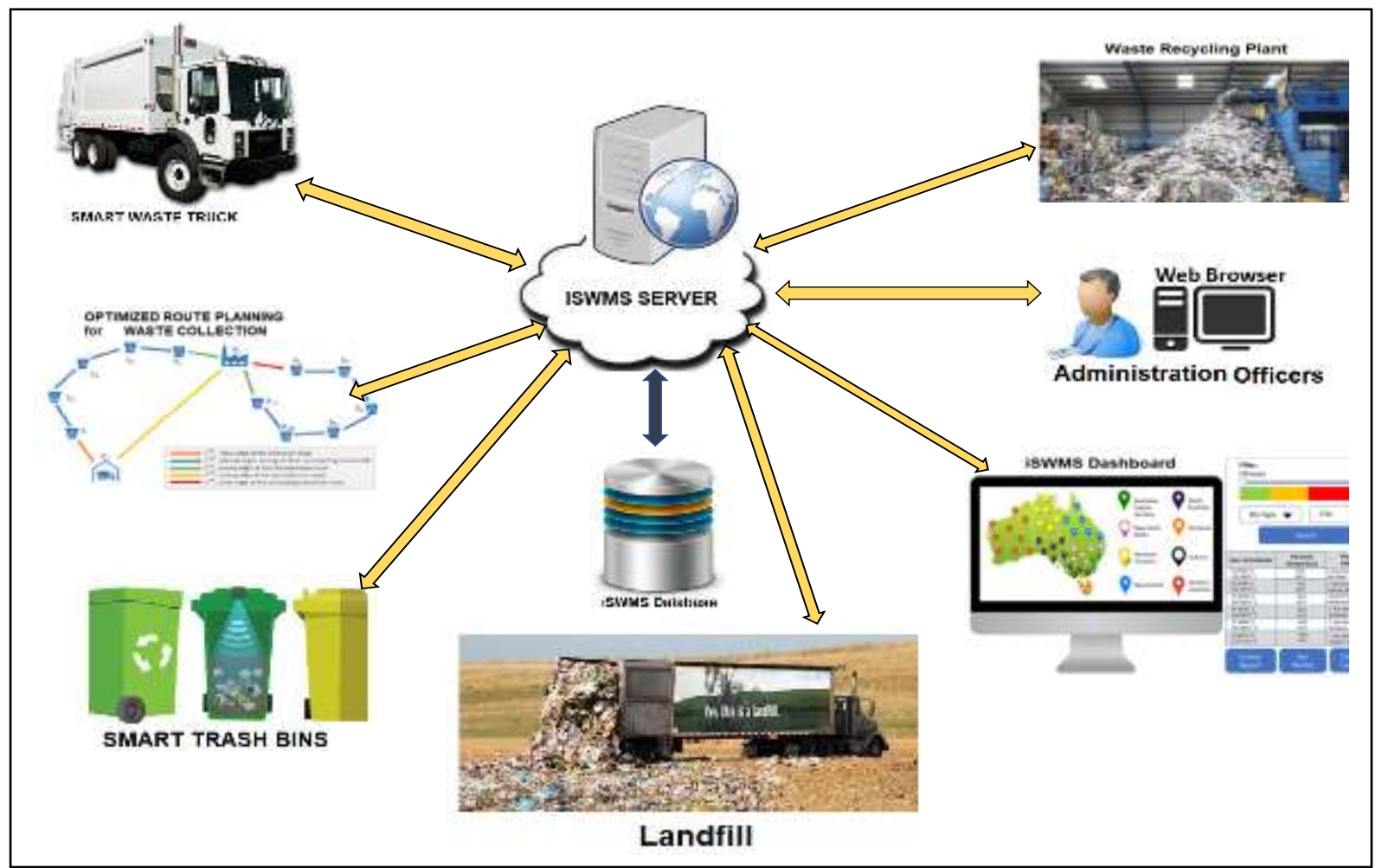

Fig. 4: High level block diagram of proposed iSmartWMS

- Load Cell: Load cell are used to identify weight of the Trash Bins. Load Cells are attached below at the bottom of Trash Bins. Weight measurement may not accurately indicate fill level of trash bin, but in case the trash bin weight reaches to the specified limit of what Garbage Truck can pick up, then waste collector vehicles can be deployed for evacuation of such trash bins. Load cells can be configured to measure weight from few kilograms to few thousand kilograms easily. Many Off the Shelf Load cells used in trash bins comes with IoT communication connectivity or with GSM Modems for communication with the external world. Such load cells are designed and manufactured to operate in harsh and wide operating temperature environment.

- Image Sensors: A network connected high resolution Camera mounted inside opening lid of trash bins in a protected IP Proof enclosure can be used to capture the trash bins images. By processing these images, it is possible to accurately know the fill status, various contents of trash bins and also the overall condition of trash bins. Image Sensors however are costly and needs special protection as well as special image processing algorithms for detecting the fill status information and condition of trash bin. The Image sensors typically find applications for trash bins which are bulky in size and storing the waste which is harmful for human beings. Some Image Sensors also provides Pan, Tilt and Zoom facility to have a closer look on the contents of bins remotely.

- Temperature and Humidity Sensors: For the trash bins storing industrial waste, needs bins servicing at regular intervals. Industrial waste is harmful, and the waste generates high temperature and causes high humidity inside trash bins over the short period of time. If not evacuated at right time such waste can impose danger to surrounding environment as well as to human beings. So, it is always necessary to detect the temperature and humidity conditions within trash bins at regular intervals. Low cost Electronic Sensors such as DHT11, DHT22 along with the controller and modem can provide the Trash bin inside temperature and humidity readings easily. However, since the sensors needs to be operated in the harsh environment, proper protection enclosure for electronic circuits is must while installing such sensors within trash bins.

- Gas Sensors: When waste such as Hospital waste, Industrial waste from chemical industries are deposited in huge trash bins, chances of generating harmful gases are very high. If the generated gas level is not checked at regular intervals, then there is a possibility of leaking out excess 
generated gas in the environment and can cause harmful effect on surrounding nature as well as human beings. Hence gas sensors are typically installed for such trash bins. The Sensor such as MQ135 is a low power and low-cost gas sensor which is sensitive to various gasses such as Methane, $\mathrm{CO}_{2}, \mathrm{NH}_{3}$, Benzene, LPG, Alcohol, and Smoke. MQ135 sensor along with Controller and Modem can provide generated gas information to control system for data analysis and needful action. If it is observed that generated gases reach to a predefine limit, then the trash bin is emptied as per standard procedure.

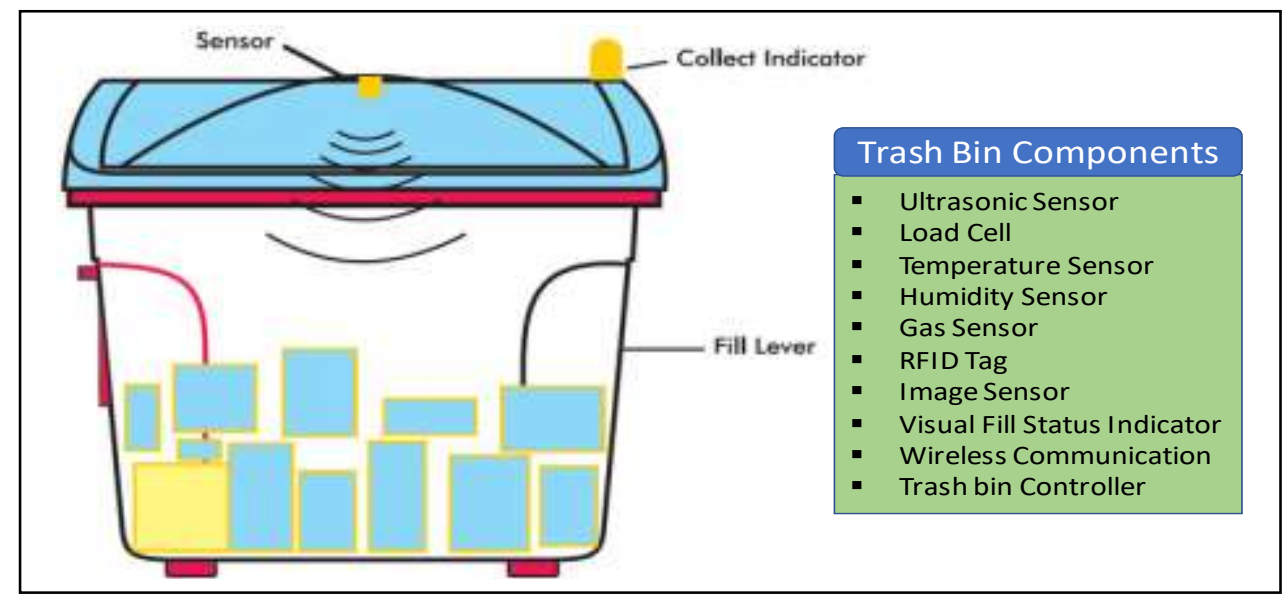

Fig. 5: Smart Trash Bin typical components

Note that data from all the installed sensors of Smart Trash Bin is communicated to the trash bin controller at regular interval. Trash Bin Controller is like a brain of Smart Trash Bin and is the only component which maintains the communication of Smart Trash Bin with the external world. The trash bin controller wirelessly communicates the consolidated data in a decided message format to the iSmartWMS Server securely. The wireless repeaters shall be used to cover appropriate connectivity range wherever required. The message from Smart Trash Bin controller is expected to contain at least following information.

$>$ Message ID: Message Identifier

$>$ Trash Bin ID: Trash Bin Identifier

$>$ Trash Bin Location Coordinates

$>$ Sensor Type: Gas/Temp/Humidity/Weight etc.

$>$ Time Stamp: Date and Time

$>$ Sensor Data: Data generated from Sensor

$>$ EOM: Message Checksum

The whole message packet then encrypted with suitable encryption algorithm such as AES128/AES256 and then communicated to iSmartWMS Server for further action. Note that the Smart Trash Bin Controller and iSmartWMS Server have two-way communications. Apart from regular data from trash bin controller, iSmartWMS Server can also request or enquire specific Sensor data from specific trash bin as per requirement. Based on such requests the trash bin controller sends the enquired sensor data to iSmartWMS Server as required.

\section{(2) Smart Waste Truck}

Smart Waste Truck (SWT) is one of the most important components in Smart Waste Management Systems. The SWTs play the most crucial role in waste management of collecting and transporting the waste to various places as per requirements. In iSmartWMS instead of the simple garbage collector, SWT is suggested to use. SWTs have following features which makes it the most recommended vehicle for garbage collection and disposal.

- Strong and robust waste container with dumpster lift

- Robotic Arm for automated waste collection 
- Facility to store wet as well as Solid waste separately

- Facility to indicate waste level

- Equipped with accelerometers, Air-quality sensors, infrared cameras, and wireless signal monitors

- Support for 24x7 secured Wireless Communication

- Installed with GPS and Client system for real time communication with Waste Management Server

SWTs can communicate with iSmartWMS Server through a secure wireless communication and can receive alerts and notifications as well as route optimization information, during travelling and while collecting waste from geographically distributed Smart Trash Bins.

\section{(3) iSmartWMS Server}

The iSmartWMS Server can be considered as brain of the entire waste management process. It is basically a dedicated physical cloud server accessed over internet remotely. The iSmartWMS Server Application is responsible for following.

- Communicate with each Smart Trash Bin Controller periodically

- Communicate with other devices such as Smart Waste Truck controller, Landfill station controller, with Administration staff over web interface, Recycling Plant Controller and with iSmartWMS Database

- Maintain communication status with all the required controllers and report communication failure if any for suitable action

- Run Smart Waste Management Application and carry out all the required data analytics on the received waste related data

- Store the Waste Management Data updates from Smart Trash bins and other controllers into the data base with appropriate timestamp

- Generate alerts and notifications in case of any emergency situations with respect to waste management

- Maintain the fill level status information of each trash bin and provide instruct to SWTs about trash bins which needs to be emptied

- Provide optimized route information to SWTs for garbage collection

- Receive the recycled material related information from Recycling plat controller and maintain such information in the database

- Receive the landfill material related information from landfill station controller and maintain such information in the database

- Generate the dashboard and maintain the real-time information about waste management providing crucial information such as

$>$ Number of filled trash bins

$>$ Number of empty trash bins

$>$ Number of underfilled trash bins

$>$ Number of trash bins which needs immediate servicing

$>$ Number of SWTs collecting waste

$>$ Garbage collection optimized route information

$>$ Total $\mathrm{Kg}$ of waste collected in a day and over a period of time

$>$ Total $\mathrm{Kg}$ of waste recycled in a day and over a period of time

$>$ Total $\mathrm{Kg}$ of waste dumped in landfill in a day and over period of time

$>$ Communication Status information with each trash bin controller

- Provide web interface to administration stakeholders and generate the necessary reports for them for offline analysis

Note that the Application Server front end and back end can be developed through Java or C\#.Net technologies. Data analytics algorithm can be developed in R or MATLAB programming language and can be subsequently integrated with the Application Server as Class Package and or through a link library. The server can be hosted on any available cloud platform such as CloudX, CloudSigma or any cost effective off the shelf cloud platform so that it can be securely accessed over internet. 


\section{(4) iSmartWMS database}

iSmartWMS database is also one of the most important elements, and it is used to store and retrieve all the waste management related data. Note that from security perspective it is recommended to store encrypted data. This database can be either build up through the open source robust and powerful databases such MySQL, MongoDB, PostgreSQL, Redis etc. and or can be implemented with one available through off the shelf cloud platform.

\section{(5) Recycling Plant Controller}

Recycling Plat Controller is responsible to provide mainly recycled material related information to the iSmartWMS Server. Recycling plant controller receives this information through a weighing scale system and or through a dedicated operator interface. Operator feeds in the required information in plant controller User Interface system on daily basis. This information is then subsequently communicated back to ISMARTWMS Server periodically and based on demand. Following information can be generated by Recycling Plant Controller.

- Received $\mathrm{Kg}$ waste material on given day

- Recycled Kg material on given day

- Total Kg waste material received over last week

- Total $\mathrm{Kg}$ waste material received so far in last one month

- Total $\mathrm{Kg}$ waste material received so far in last one year

- Total Kg material recycled over last week

- Total Kg material recycled so far in last one month

- Total $\mathrm{Kg}$ material recycled so far in last one year

- Recycled Items information

- Information about Normal operation/ Alert/Emergency Situation

- Health Status information

\section{(6) Landfill Station Controller}

Landfill Controller is responsible to provide mainly landfilled material related information to the iSmartWMS Server. Similar to Recycling plant controller, Landfill Controller too receives the landfill material related information through a weighing scale system which is used to measure the weight of the deposited waste and or through a dedicated operator interface. Operator feeds in the required information in Landfill Controller User Interface system on daily basis. This information is then subsequently communicated back to iSmartWMS Server periodically and based on demand.

Following information can be generated by Landfill Station Controller.

- Received Kg waste material on given day

- Total Kg waste material landfilled over last week

- Total Kg waste material landfilled so far in last one month

- Total $\mathrm{Kg}$ waste material landfilled so far in last one year

- Information about Generated/emitted Gas on given day

- Information about Normal operation/ Alert/Emergency Situation

- Health Status information

\section{RESULTS AND DISCUSSION :}

In this paper the iSmartWMS system is mainly discussed at concept level. Due to time and budget constraints, it was not possible to implement the iSmartWMS system in totality. However, proof of concept of iSmartWMS is developed with limited functionality to test at laboratory level environment. Simulated data with respect to waste collection was generated and tested with iSmartWMS Server to check its data analytics related performance, ability to generate alerts and notifications with respect to emergency situations and or situations where any trash bin needs urgent service. By using google map of a specific geography and simulated trash bin data, iSmartWMS SWT route Optimization data generation capability was also tested successfully. Finally, through web interface a secure access was provided with ISMARTWMS server for authenticated users and dashboard related data display capability of ISMARTWMS server was tested A simple user-friendly front-end User interface is 
developed with C\# for the iSmartWMS Server Application configurations and reporting. For database, I used the MySQL which proved to be simple but very effective for this application. Since the system was tested with limited simulated data, the full potential and capability of iSmartWMS system could not be tested. However, overall, the iSmartWMS system worked well. The results found very encouraging. The User feedback was collected during lab testing and many users appreciated the developed solution.

\section{CONCLUSION :}

The testing of POC of iSmartWMS System successfully demonstrated that iSmartWMS solution effectively carried out all the intended functionalities. Due to limited test data and in absence of reallife waste material related data, full potential of iSmartWMS PoC could not be tested and it is difficult to judge the overall performance of iSmartWMS as a Smart Waste management system. However, the encouraging results confirmed that iSmartWMS can be effectively used for Smart Waste Management if implemented fully. To take full advantage of the benefits offered by iSmartWMS, it is highly recommended to develop the full-fledged system as per stated requirements and building blocks.

\section{FUTURE WORK :}

Based on the encouraging results obtained through verification and validation of iSmartWMS PoC, it is recommended to develop and implement full-fledged iSmartWMS system. It is also recommended to integrate it with any available Smart City infrastructure and validated against real life waste generated data. During laboratory testing of iSmartWMS PoC some users provide valuable suggestions as follows.

- Like web interface, provide iSmartWMS access over smartphone for easy and convenient access for authorized administrative staff. This will provide more flexibility to admin staff for using and maintaining the system.

- Apart from visual indication, audio alarm should also be provided periodically if any trash been need immediate servicing.

- Provide facility to integrate iSmartWMS System software with the Smart City existing IT Infrastructure

- Facility to view Smart Trash bin contents video footage to authorized users

Considering the usefulness and value lies in above listed suggestions, it is recommended to consider the above listed improvements as well while implementing the iSmartWMS based Smart Waste Management Solution.

\section{REFERENCES :}

[1] Kodwo Miezaha, Kwasi Obiri-Dansoa, Zsófia Kádárc, Bernard Fei-Baffoea, et al. (2015). Municipal solid waste characterization and quantification as a measure towards effective waste management in Ghana. Elsevier, Waste Management, 46(1), 15-27.

[2] Kellow Pardini, Joel J.P.C. Rodrigues, Ousmane Diallo, et al. (2020). A Smart Waste Management Solution Geared towards Citizens. Sensors, 20(1), 1-15.

[3] Pedro Branco, liKim Bolton, Mattias Eriksso. (2020). Environmental impacts of waste management and valorisation pathways for surplus bread in Sweden. Waste Management, 117(1), $136-145$.

[4] Ian Tiseo (2020). Global waste generation outlook by region 2016-2050. https://www.statista.com/statistics/233613/waste-generation-worldwide-by-region/. Accessed on $29 / 04 / 2021$

[5] Introduction Types of Waste - ESCAP. https://www.unescap.org/sites/default/files/CH08.PDF. Accessed on 29/04/2021.

[6] Muncipal Solid Waste Management Manual Part II (2020). Central Public Health and Environmental Engineering Organisation (CPHEEO). Ministry of Urban Development, Government of India. 3-41. 
[7] Dr. Dipak Gade. (2019). Introduction to Smart Cities and Selected Literature Review. International Journal of Advance and Innovative Research, 6(2), Part - 4. 7-19.

[8] Insung Hong, Sunghoi Park et al. (2014). IoT-Based Smart Garbage System for Efficient Food Waste Management. Hindawi, The Scientific World Journal, 2014(1). 1-14.

[9] María-Victoria Bueno-Delgado, José-Luis Romero-Gázquez, et al. (2019). Optimal Path Planning for Selective Waste Collection in Smart Cities. Sensors, 19(1). 1-14.

[10] Yann Glouche, Paul Couderc. (2013). A Smart Waste Management with Self-Describing objects, The Second International Conference on Smart Systems, Devices and Technologies, IARIA. 6370.

[11] Zeki Oralhan, Burcu Oralhan, and Yavuz YİĞíT. (2016). Smart City Application: Internet of Things (IoT) Technologies Based Smart Waste Collection Using Data Mining Approach and Ant Colony Optimization. The International Arab Journal of Information Technology, 14(4). 423-427.

[12] Popa, C. L., Carutasu, G., Cotet, C. E., Carutasu, N. L., \& Dobrescu, T. (2017). Smart city platform development for an automated waste collection system. Sustainability, 9(11), 1-15.

[13] Dr. Dipak Gade. (2019). Technology Trends and Digital Solutions for Smart Cities Development. International Journal of Advance and Innovative Research, 6(1), Part - 4. 29-37.

[14] Eunice David Likotiko, Devotha Nyambo, Joseph Mwangoka. (2017). Multi-agent based IOT Smart Waste Monitoring and Collection Architecture. International Journal of Computer Science, Engineering and Information Technology, 7(5).1-14.

[15] Behzad Esmaeiliana, Ben Wangb, Kemper Lewisc, et al. (2018). The future of waste management in smart and sustainable cities: A review and concept paper. Elsevier, Waste Management, 81. 177-195.

[16] Kellow Pardini et al. (2019). IoT-Based Solid Waste Management Solutions: A Survey. Journal of Sensor and Actuator Networks, 8(1). 1-25.

[17] Florin-Constantin Mihai and Mohammad J. Taherzadeh. (2017). Introductory Chapter: Rural Waste Management Issues at Global Level. Intech, Book: Solid Waste Management in Rural Areas. 1-12.

[18] The Impact of the 4th Industrial Revolution on the Waste Management Sector. https://www.iswa.org/knowledge-base/the-impact-of-the-4th-industrial-revolution-on-the-wastemanagement-sector/?v=c86ee0d9d7ed. Accessed on 29/04/2021.

[19] Landfill Gas to Energy Turning waste into energy. https://www.advanceddisposal.com/formother-earth-2/education-zone/landfill-gas-to-energy.aspx. Accessed on 29/04/2021.

[20] Alexey Medvedev, Petr Fedchenkov, Arkady Zaslavsky, et al. (2015). Waste management as an IoT enabled service in Smart Cities. Springer, 1. 1-13.

[21] Tran Anh Khoa, Cao Hoang Phuc, et al. (2019). Waste Management System Using IoT-Based Machine Learning in University. Wireless Communications and Mobile, Computing, 2020(1). 113.

[22] Asim Zeb, Qurban Ali, Muhammad Qaiser Saleem. (2018). A Proposed IoT-Enabled Smart Waste Bin Management System and Efficient Route Selection. Journal of Computer Networks and Communications, 2019(1). 1-9.

[23] Sani Abba and Chinaka Ihechukwu Light. (2020). IoT-Based Framework for Smart Waste Monitoring and Control System: A Case Study for Smart Cities. Engineering Proceedings, 2(1), $1-10$.

[24] Gianni Minetti (2020). Smart waste, an opportunity cities should not trash. https://www.smartcitiesworld.net/. Accessed on 29/04/2021. 
[25] Theodoros Anagnostopoulos, Arkady Zaslavsky, Kostas Kolomvatsos, et al. (2017). Challenges and Opportunities of Waste Management in IoT-Enabled Smart Cities: A Survey. IEEE Transactions on Sustainable Computing, 2(1). 275-289. 